\title{
EMBEDDING IN TOPOLOGICAL VECTOR SPACES
}

\author{
GARY RICHARDSON
}

\begin{abstract}
Let $L X$ denote the set of all continuous linear functionals on the locally convex topological vector space $X$. The space $L_{c_{0} X}$ denotes $L X$ endowed with the compact-open topology. We investigate the spaces, $X$, which have the property that the natural map from $X$ into $L_{\mathrm{co}}\left(L_{\mathrm{co}} X\right)$ is an embedding.
\end{abstract}

Preliminaries. The reader is referred to Jarchow [6] for basic definitions and terminology not mentioned here. Since convergence spaces are more abundant than topological spaces, it is sometimes convenient to characterize certain topological properties in terms of an associated convergence space. For example, the completion of a Hausdorff locally convex topological vector space $X$ is shown by Butzmann [1] to be $L_{c}\left(L_{c} X\right)$, where $L_{c} X$ denotes the set $L X$ endowed with the continuous convergence structure; that is, the finest convergence structure, $c$, such that the evaluation map $\omega: L_{c} X \times X \rightarrow R$ is continuous. Convergence space properties of $L_{c} X$ are used here to investigate the spaces, $X$, such that the natural map $i: X \rightarrow L_{\text {co }}\left(L_{\text {co }} X\right)$ is an embedding, where "co" denotes the compact-open topology. A space $X$ satisfying the latter condition is called co-embedded.

The symbol $X$ will always denote a Hausdorff locally convex topological vector space. Another convenient convergence vector space associated with $X$ is $X^{\wedge}$, defined as the set $X$ equipped with the following convergence structure: $\mathscr{F} \rightarrow x$ in $X^{\wedge}$ iff $\mathscr{F} \rightarrow x$ in $X$ and $\mathscr{F}$ contains an $X$-compact subset. The space $X^{\wedge}$ is the coarsest locally compact convergence vector space finer than $X$. Moreover, for any convergence vector space $Y$, let $K Y$ denote the finest locally convex topological vector space coarser than $Y$. Let $k X$ denote the coarsest $k$-space finer than $X$, and let $c k X$ be the space obtained from $k X$ by taking the convex neighborhoods of 0 in $k X$ as a base for the neighborhood filter of 0 in $c k X$. These notions were introduced and studied by Frölicher and Jarchow [3]. A space $X$ is called a ck-space whenever $c k X=X$. Moreover, $c k X$ is the finest locally convex topological vector space which has the same compact subsets as $X$; furthermore, $K X^{\wedge}=c k X$. Hence ti follows that $X$ is a $c k$-space iff $X=K Y$ for some locally compact convergence vector space

Presented to the Society, April 25, 1976 under the title Co-embedded spaces; received by the editors August 20, 1976 and, in revised form, February 18, 1977.

AMS (MOS) subject classifications (1970). Primary 54A20, 54C25, 46A05.

Key words and phrases. Topological vector spaces, convergence spaces, continuous convergence.

O American Mathematical Society 1977 
$Y$. Butzmann [1] has shown that $L_{c} X$ is a locally compact convergence vector space.

The notation $L_{\mathrm{co}}\left(L_{\mathrm{co}} X\right)$ is condensed to simply $L_{\mathrm{co}}^{2} X$; similarly for $L_{c}\left(L_{c} X\right)$. Thus $X$ is co-embedded iff $i: X \rightarrow L_{\text {co }}^{2} X$ is an embedding. Cook and Fischer [2] have shown that the equicontinuous subsets of $L X$ and the relatively compact subsets of $L_{c} X$ coincide. Frölicher and Jarchow [3] have shown that the equicontinuous subsets of $L X$ coincide with the relatively compact subsets of $L_{\text {co }} X$ iff $X$ is co-embedded; in particular, barrelled spaces and $c k$-spaces are co-embedded. It is shown later that a co-embedded space is not necessarily a $c k$-space. It is straightforward to show that if $X$ and $X^{\wedge}$ have the same duals, then $X$ is co-embedded iff $X$ is a $c k$-space.

2. Co-embedded spaces. Let $\tilde{X}$ denote the completion of $X$. It follows from Grothendieck's completeness theorem that if $X$ is co-embedded, then $L_{\text {co }}^{2} X$ is a subspace of $\tilde{X}$; in particular, $L_{\text {co }}^{2} X$ is a subspace of $L_{c}^{2} X$.

Proposition 2.1. A space $X$ is co-embedded iff $\left(L_{\mathrm{co}} X \hat{)}=L_{c} X\right.$.

Proof. As mentioned in $\S 1$, the equicontinuous subsets and the relatively compact subsets of $L_{c} X$ coincide. Since $L_{c o} X$ and $\left(L_{\mathrm{co}} X\right)$ have the same compact subsets, then $X$ is co-embedded. Conversely, suppose that $L_{\text {co }} X$ and $L_{c} X$ have the same compact subsets. It is shown by Jarchow [7] that $L_{c} X$ is the convergence space inductive limit of the spaces $\left\{\left(U^{\circ}, \sigma_{U}\right) \mid U\right.$ is a neighborhood of 0 in $X$ \}, where $U^{\circ}$ is the polar of $U$ in $L X$ and $\sigma_{U}$ is the topology of pointwise convergence. Thus $\left(L_{\mathrm{co}} X \hat{)}=L_{c} X\right.$ follows immediately.

Let $\Lambda Y$ denote the finest topological space which is coarser than the convergence space $Y$.

Proposition 2.2. Suppose that $X$ is co-embedded; then $\Lambda L_{c} X\left(K L_{c} X\right)$ is the only $k(c k)$-space between $L_{\mathrm{co}} X$ and $L_{c} X$. Further, if $L_{\mathrm{co}} X$ and $L_{c} X$ have the same duals, then $K L_{c} X$ is the only co-embedded space between $L_{\mathrm{co}} X$ and $L_{c} X$.

Proof. Let $Y$ be a $k$-space such that $L_{\text {co }} X \leqslant Y \leqslant L_{c} X$. From Proposition 2.1, $Y^{n}=L_{c} X$, and since $Y$ is a $k$-space, then by Theorem 3.1 of [8], $\Lambda Y^{n}=Y$, so $\Lambda L_{c} X=Y$. Next, let $Y$ be a co-embedded space such that $L_{\mathrm{co}} X \leqslant Y \leqslant$ $L_{c} X$. By hypothesis, it follows that $L_{\mathrm{co}}^{2} Y=L_{\mathrm{co}}^{2}\left(K L_{c} X\right)$, so $Y=K L_{c} X$.

Corollary 2.3. Let $X$ be a co-embedded space such that $L_{\mathrm{co}} X$ and $L_{c} X$ have the same duals. Then $L_{\mathrm{co}} X$ is co-embedded iff it is a ck-space.

Komura [9] has given an example of a Montel space $X$ which is not complete. It follows that $L_{\beta} X=L_{\mathrm{co}} X$, where $\beta$ denotes the strong topology. Since $L_{\beta} X$ is also a Montel space, then $X=L_{\beta}^{2} X \neq \tilde{X}$. Moreover, since $X$ is co-embedded, then $L_{\mathrm{co}}^{2} X$ is a subspace of $\tilde{X}$, so it follows that $L_{\mathrm{co}} X$ and $L_{c} X$ do not have the same duals; in particular $K L_{c} X \neq L_{\mathrm{co}} X$. Hence it follows from Proposition 2.1 and $\S 1$ that $L_{\mathrm{co}} X$ is not a $c k$-space. This shows that the duality condition in Corollary 2.3 cannot be eliminated since $L_{\mathrm{co}} X$ is co-embedded yet not a $c k$-space. 
In case $X$ is metrizable, then by the Banach-Dieudonne' Theorem (cf. [5, p. 272 (1) and (3))], it follows that $L_{\mathrm{co}} X=\Lambda L_{c} X$. Hence $L_{\mathrm{co}} X$ is a $c k$-space and has the same dual as $L_{c} X$, so $L_{\mathrm{co}} X$ is co-embedded and also $L_{\mathrm{co}}^{2} X=\tilde{X}$.

Proposition 2.4. Let $X$ be a space such that $i(X)$ is dense in $L_{\mathrm{co}}^{2} X$. Then $i_{L X}: L_{\mathrm{co}} X \rightarrow L_{\mathrm{co}}^{3} X$ is an onto embedding iff $L_{\mathrm{co}} X$ is co-embedded.

Proof. Suppose that $i_{L X}: L_{\mathrm{co}} X \rightarrow L_{\mathrm{co}}^{3} X$ is an embedding. Since $i_{X}(X)$ is dense in $L_{\mathrm{co}}^{2} X$, then it follows that $i_{X}^{*}: L_{\mathrm{co}}^{3} X \rightarrow L_{\mathrm{co}} X$ is injective. Moreover, $i_{X}^{*} \circ i_{L X}$ is the identity map on $L X$. Hence $i_{L X}$ is an onto embedding.

Corollary 2.5. Suppose that $i_{X}: X \rightarrow L_{\mathrm{co}}^{2} X$ is an onto function. Then $i_{L X}$ : $L_{\mathrm{co}} X \rightarrow L_{\mathrm{co}}^{3} X$ is an onto embedding.

Proof. Since $i_{X}^{-1}: L_{\mathrm{co}}^{2} X \rightarrow X$ is continuous, then $\left(i_{X}^{-1}\right)^{*}: L_{\mathrm{co}} X \rightarrow L_{\mathrm{co}}^{3} X$ is also continuous. However, it is straightforward to check that $\left(i_{X}^{-1}\right)^{*}=i_{L X}$, so by Proposition $2.4, i_{L X}$ is an onto embedding.

Some of the above results give sufficient conditions in order that $L_{\mathrm{co}} X$ be co-embedded. However, this question remains, in general, unanswered. Our next proposition deals with the existence of a coarsest co-embedded space which is finer than a given space.

LEMMA 2.6. Let $Y$ be any co-embedded space which is finer than $X$; then $Y \geqslant i_{X}(X)$, where the latter is considered as a subspace of $L_{\mathrm{co}}^{2} X$.

Proof. Let $\alpha: Y \rightarrow X$ denote the identity map. Then the following diagram is commutative and $\alpha^{* *} \circ i_{y}$ is continuous.

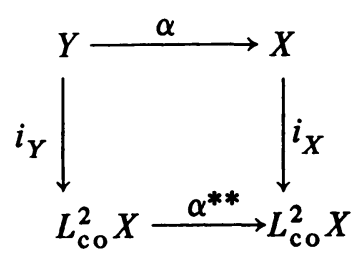

Hence it follows that $Y \geqslant i_{X}(X)$.

Let $X$ be a given space and define $X_{\beta}$ recursively as follows: $X_{0}=X$; $X_{\beta}=i\left(X_{\beta-1}\right)$ whenever $\beta$ is a nonlimit ordinal $\geqslant 1 ; X_{\beta}=\sup \left\{X_{\alpha} \mid \alpha<\beta\right\}$ whenever $\beta$ is a limit ordinal. Note that each $X_{\beta}$ is a Hausdorff locally convex topological vector space and that $X \leqslant X_{\alpha} \leqslant X_{\beta}$ whenever $\alpha \leqslant \beta$. The chain $\left\{X_{\beta} \mid \beta \geqslant 0\right\}$ must eventually terminate for some $\beta$. Hence $X_{\beta+1}=X_{\beta}$ is a co-embedded space. From Lemma 2.6, it follows that $X_{\beta}$ is the coarsest co-embedded space which is finer that $X$, and is denoted by co $X$. By Theorem 3.3 and Corollary 3.4 of Frölicher and Jarchow [3], it follows that if $X$ is complete then $\operatorname{co} X$ is also complete. Hence we have shown the following. 
Proposition 2.7. There is a coarsest co-embedded space, co $X$, finer than $X$. Moreover, if $X$ is complete, then co $X$ is also complete.

Haydon [4] has given an example of a complete space $X$ such that $c k X$ is not complete. Hence it follows from Proposition 2.7 that, in general, co $X$ and $c k X$ differ. Since $c k X$ is co-embedded, then $c k X \geqslant$ co $X$ holds in general.

The following interesting result was pointed out to me by Professor Kelley McKennon at the American Mathematical Society meeting in Reno, Nevada.

Proposition 2.8. An infrabarrelled space $X$ is co-embedded.

Proof. Let $A$ be a compact subset of $L_{\text {co }} X$ and suppose that $A$ is not strongly bounded. Then there exists a bounded subset $\left\{x_{n} \mid n \geqslant 1\right\}$ of $X$ and a subset $\left\{f_{n} \mid n \geqslant 1\right\}$ of $A$ such that for each natural number $n, f_{n}\left(x_{n}\right)>2 n$. Let $\Phi$ be an ultrafilter on $L X$ containing the filter generated by the sequence $\left(f_{n}\right)$. Since $A$ is compact, then assume that $\Phi$ converges to $f$ in $L_{\text {co }} X$. Further, since $\left(x_{n} / n\right)$ converges to 0 in $X$, then $\Phi$ converges uniformly to $f$ on the compact subset $K=\left\{x_{n} / n, 0 \mid n \geqslant 1\right\}$ of $X$. Let $H \in \Phi$ such that $|g(z)-f(z)| \leqslant 1$ for each $g \in H$ and $z \in K$. Let $\left(f_{n_{k}}\right)$ be a subsequence of $\left(f_{n}\right)$ contained in $H$. Since $\left|f\left(x_{n_{k}} / n_{k}\right)\right| \leqslant 1$ for $k$ sufficiently large, then it follows that $\left|f_{n_{k}}\left(x_{n_{k}}\right)\right| \leqslant$ $2 n_{k}$ for $k$ sufficiently large, which is contrary to $f_{n}\left(x_{n}\right)>2 n$ for all $n \geqslant 1$. Hence $A$ is strongly bounded, and so by hypothesis $A$ is equicontinuous.

Concluding Remarks. It can be shown by using routine arguments that the property of being co-embedded is preserved under the process of taking locally convex direct sums, a continuous open linear image, inductive limits, and products. Furthermore, it can be shown that if $X$ is co-embedded, then its completion $\tilde{X}$ is also co-embedded; in fact, $i_{\tilde{X}}: \tilde{X} \rightarrow L_{\mathrm{co}}^{2} \tilde{X}$ is an onto embedding since $L_{\text {co }}^{2} \tilde{X}$ is complete.

Note that Corollary 2.5 implies that if $i: X \rightarrow L_{\mathrm{co}}^{2} X$ is an onto function, as is the case whenever $X$ is complete, then co $X=L_{\mathrm{co}}^{2} X$. Moreover, the example given in $\S 6.1$ of [3] is complete but not co-embedded. However, if $X$ is complete and has the Mackey topology $\tau(X, L X)$, then it follows easily that $X$ is co-embedded. Since there are $c k$-spaces which do not have the Mackey topology (cf. $\$ 6.2$ of [3]), then co-embedded spaces do not necessarily have the Mackey topology. We conclude with the following question. Is a space endowed with the Mackey topology necessarily co-embedded?

\section{REFERENCES}

1. H. P. Butzmann, Über die c-Reflexivität von $C_{c}(X)$, Comment. Math. Helv. 47 (1972), 92-101.

2. C. H. Cook and H. R. Fischer, On equicontinuity and continuous convergence, Math. Ann. 159 (1965), 94-104.

3. A. Frölicher and H. Jarchow, Zur Dualitätstheorie kompakt erzeugter und lokalkonvexer Vektorräume, Comment. Math. Helv. 47 (1972), 289-310.

4. R. Haydon, Sur un probleme de H. Buchwalter, C. R. Acad. Sci. Paris Ser. A-B 275 (1972), A1077-A1080. 
5. G. Köthe, Topological vector spaces. I, Springer-Verlag, Berlin and New York, 1969.

6. H. Jarchow, Marinescu-Räume, Comment. Math. Helv. 44 (1969), 138-163.

7. __ Duale Charakterisierungen der Schwartz-Räume, Math. Ann. 196 (1972), 85-90.

8. D. Kent and G. Richardson, Locally compact convergence spaces, Michigan Math. J. 22 (1975), 353-360.

9. Y. Komura, Some examples on linear topological spaces, Math. Ann. 153 (1964), 150-162.

Department of Mathematics, East Carolina University, Greenville, North Carolina 27834 